Research Article

\title{
Homeomorphism and Quotient Mappings in Infrasoft Topological Spaces
}

\author{
Tareq M. Al-shami iD \\ Department of Mathematics, Sana'a University, Sana'a, Yemen \\ Correspondence should be addressed to Tareq M. Al-shami; tareqalshami83@gmail.com
}

Received 16 July 2021; Accepted 29 July 2021; Published 10 August 2021

Academic Editor: Huseyin Isik

Copyright (C) 2021 Tareq M. Al-Shami. This is an open access article distributed under the Creative Commons Attribution License, which permits unrestricted use, distribution, and reproduction in any medium, provided the original work is properly cited.

In this paper, we contribute to infrasoft topology which is one of the recent generalizations of soft topology. Firstly, we redefine the concept of soft mappings to be convenient for studying the topological concepts and notions in different soft structures. Then, we introduce the concepts of open, closed, and homeomorphism mappings in the content of infrasoft topology. We establish main properties and investigate the transmission of these concepts between infrasoft topology and its parametric infratopologies. Finally, we define a quotient infrasoft topology and infrasoft quotient mappings and study their main properties with the aid of illustrative examples.

\section{Introduction}

We face vagueness, ambiguity, and representation of imperfect knowledge in different areas such as economics, engineering, medical science, sociality, and environmental sciences. Mathematicians, engineers, and scientists, particularly those who focus on artificial intelligence, are seeking for approaches to solve the problems that contain vagueness. But they experienced a trouble: how they can formulate uncertain concepts that may not involve mathematically definite results. This means that there is a need for alternative mathematical concepts. Therefore, they have begun to look for different fields of research which leads to initiate several set theories as an alternative to George Cantor's set theory such as fuzzy set (and its generalizations such as intuitionistic fuzzy set and pythagorean fuzzy set), rough set, multiset, and recently soft set.

Soft set was proposed by Molodtsov [1] as one of the nonstatistical mathematical approaches targeting to deal with ambiguous, undefined, and imprecise meaning. It is characterized by flexibility and fruitful applications compared with other uncertainty theories. Since Molodtsov put forward the concept of soft sets, many scholars have applied it in several research areas such as decision-making problems [2], systems of linear equations [3], computer science [4], engineering [5], and medical sciences [6].
The year 2011 was the beginning point of the interaction between soft set theory and topology. Simultaneously, Shabir and $\mathrm{Naz}$ [7] and Çağman et al. [8] initiated the concept of soft topology. However, they used different techniques to formulate soft topology. On the one hand, Shabir and Naz formulated soft topology on the collection of soft sets over a universal crisp set and a fixed set of parameters. On the other hand, Çağman et al. formulated soft topology on the collection of soft sets over an absolute soft set and different sets of parameters. We conduct this study in the frame of Shabir and Naz's definition which is more analogous to the classical topology.

Kharal and Ahmad [9] defined soft mappings using two ordinary (crisp) mappings, one of them between the sets of parameters and the other between the universal sets. However, we reformulate this definition using the concept of soft points to be convenient for studying in different soft structures. We classify soft mappings by soft spaces into different families such as continuous, open, closed, and homeomorphism mappings. In addition, soft mappings enable us to classify topological concepts in terms of preservation under specific classes of soft mappings. In [10], the authors presented another point of view to study soft mappings with a medical application. Zorlutuna and Çakir [11] investigated continuity between soft topological spaces. The authors of [12] presented new relations between ordinary points and soft sets to define new types of soft 
separation axioms. Quite recently, Al-shami and Kočinac [13] investigated the conditions under which some concepts are kept between soft topology and its parametric topologies. Kočinac et al. [14] discussed selection principles in the context of soft sets.

Some generalizations of soft topology were introduced and studied. For example, El-Sheikh and Abd El-Latif [15] established the concept of suprasoft topological spaces by neglecting a finite intersection condition of a soft topology. Thomas and John [16] formulated the concept of soft generalized topological spaces, and Zakari et al. [17] originated the concepts of soft weak structures. Lately, Al-shami et al. [18] have initiated and investigated the concept of infrasoft topology. Then, Al-shami [19] studied infrasoft compactness and its application to fixed point theorem. Also, Al-shami and Abo-Tabl [20] defined the concepts of infrasoft connected and infrasoft locally connected spaces. As a continuation of this work, we conduct this study. We aim to redefine soft mappings and explore some types of them through the infrasoft topology content.

We organize this article as follows. After the introduction section, Section 2 mentions some concepts and notions that clarify the investigations of this paper. In Section 3, we show shortcoming of soft mapping defined in [9] and reformulate it simulating to the definition of (crisp) mappings. In Section 4, we define new types of infrasoft mappings and investigate main properties. Among other things, we prove that these infrasoft mappings are preserved under product of infrasoft topological spaces and investigate the transmission of these concepts between infrasoft topology and its parametric infratopologies. We devote Section 5 to study quotient topology and mappings in the content of infrasoft topological spaces. Finally, we outline the fundamental obtained results and suggest some upcoming works in Section 6.

\section{Preliminaries}

In this section, we mention the main concepts that make this paper self-contained.

Definition 1 (see [1]). We called a pair $(H, A)$ a soft set over the universal set $X \neq \varnothing$ with a set of parameters $A$ provided that $H$ is a mapping from $A$ to the power set $2^{X}$ of $X$. We usually write a soft set $(H, A)$ as follows $(H, A)=\left\{(a, H(a)): a \in A\right.$ and $\left.H(a) \in 2^{X}\right\}$. The symbol $S\left(X_{A}\right)$ denotes the set of all soft sets over $X$ with any subset of $A$.

We recall some special types of soft sets.

(i) If $H(a)=\varnothing$ for each $a \in A$, then $(H, A)$ is called a null soft set [21].

(ii) If $H(a)=X$ for each $a \in A$, then $(H, A)$ is called an absolute soft set [21].

(iii) If $H(a)=\{x\}$ and $H\left(a^{\prime}\right)=\varnothing$ for each $a^{\prime} \in A \backslash\{a\}$, then $(H, A)$ is called a soft point. It is denoted by $P_{a}^{x}$. The set of all soft points over $X$ with $A$ is denoted by $P\left(X_{A}\right)$. We say that $P_{a}^{x}$ belongs to a soft set $(F, A)$, denoted by $P_{a}^{x} \in(F, A)$ if $x \in F(a)$ [22]. More details concerning soft points were given in [23].
Definition 2 (see [24]). A soft set $\left(H^{c}, A\right)$ is called a relative complement of a soft set $(H, A)$ provided that a mapping $H^{c}: A \longrightarrow 2^{X}$ is defined by $H^{c}(a)=X \backslash H(a)$ for each $a \in A$.

Since an infrasoft topological space is defined under a constant set of parameters, we will recall the previous definitions and results under a constant set of parameters.

Definition 3 (see $[21,24,25])$. Let $(F, A)$ and $(H, A)$ be two soft sets over $X$.

(i) $(F, A)$ is a soft subset of a soft set $(H, A)$, symbolized by $(F, A) \sqsubseteq(H, A)$, if $F(a) \subseteq H(a)$ for all $a \in A$.

$(F, A)$ and $(H, A)$ are called soft equal if $(F, A) \sqsubseteq(H, A)$ and $(H, A) \sqsubseteq(F, A)$.

(ii) The soft intersection of $(F, A)$ and $(H, A)$, symbolized by $(F, A) \sqcap(H, A)$, is a soft set $(M, A)$ such that a mapping $M: A \longrightarrow 2^{X}$ is given by $M(a)=$ $F(a) \cap H(a)$ for each $a \in A$.

(iii) The soft union of $(F, A)$ and $(H, A)$, symbolized by $(F, A) \sqcup(H, A)$, is a soft set $(M, A)$ such that a mapping $M: A \longrightarrow 2^{X}$ is given by $M(a)=$ $F(a) \cup H(a)$ for each $a \in A$.

More details concerning soft intersection and union were given in [26].

Definition 4 (see [27]). The Cartesian product of $(H, A)$ and $(F, B)$, which are defined over $X$ and $Y$, respectively, is a soft set, denoted by $(H \times F, A \times B)$, given by $(H \times F)(a, b)=$ $H(a) \times F(b)$ for each $(a, b) \in A \times B$.

Definition 5 (see [9]). Let $g: X \longrightarrow Y$ and $\varphi: A \longrightarrow B$ be two crisp mappings. Then, a soft mapping $g_{\varphi}: S\left(X_{A}\right)$ $\longrightarrow S\left(Y_{B}\right)$ is defined as follows: the image of a soft set $(H, M)$ in $S\left(X_{A}\right)$ is a soft set $g_{\varphi}(H, M)=(g(H), E)$ in $S\left(Y_{B}\right)$ such that $E=\varphi(M) \subseteq B$, and a mapping $g(H)$ is given by

$$
g(H)(e)=g\left(\underset{\lambda \in \varphi^{-1}(e) \cap M}{\cup} H(\lambda)\right), \quad \text { for each } e \in E .
$$

Definition 6 (see [9]). Let $g_{\varphi}: S\left(X_{A}\right) \longrightarrow S\left(Y_{B}\right)$ be a soft mapping. Then, the preimage of a soft set $(U, N)$ in $S\left(Y_{B}\right)$ is a soft set $g_{\varphi}^{-1}(U, N)=\left(g^{-1}(U), D\right)$ in $S\left(X_{A}\right)$ such that $D=\varphi^{-1}(N) \subseteq A$, and a mapping $g^{-1}(U)$ is given by

$$
g^{-1}(U)(d)=g^{-1}(U(\varphi(d)), \quad \text { for each } d \in D \text {. }
$$

Definition 7 (see [9]). We call $g_{\varphi}: S\left(X_{A}\right) \longrightarrow S\left(Y_{B}\right)$ an injective (resp. a surjective, a bijective) soft mapping if $g$ and $\varphi$ are injective (resp. surjective, bijective) mappings.

Proposition 1 (see [9]). Consider $g_{\varphi}: S\left(X_{A}\right) \longrightarrow S\left(Y_{B}\right)$ is a soft mapping. Let $\left(H_{1}, A\right)$ and $\left(H_{2}, A\right)$ be two soft sets in $S\left(X_{A}\right)$ and $\left(U_{1}, B\right)$ and $\left(U_{2}, B\right)$ be two soft sets in $S\left(Y_{B}\right)$. Then, we have the following results: 
(i) If $\left(H_{1}, A\right) \sqsubseteq\left(H_{2}, A\right)$, then $g_{\varphi}\left(H_{1}, A\right) \sqsubseteq g_{\varphi}\left(H_{2}, A\right)$

(ii) If $\left(U_{1}, B\right) \sqsubseteq\left(U_{2}, B\right)$, then $g_{\varphi}^{-1}\left(U_{1}, B\right) \sqsubseteq g_{\varphi}^{-1}\left(U_{2}, B\right)$

(iii) $\left(H_{1}, A\right) \sqsubseteq g_{\varphi}^{-1} g_{\varphi}\left(H_{1}, A\right)$ and the equality relation holds if $g_{\varphi}$ is injective

(vi) $g_{\varphi} g_{\varphi}^{-1}\left(U_{1}, B\right) \sqsubseteq\left(U_{1}, B\right)$ and the equality relation holds if $g_{\varphi}$ is surjective

(v) $g_{\varphi}\left[\left(H_{1}, A\right) \sqcup\left(H_{2}, A\right)\right]=g_{\varphi}\left(H_{1}, A\right) \sqcup g_{\varphi}\left(H_{2}, A\right)$

(vi) $g_{\varphi}\left[\left(H_{1}, A\right) \sqcap\left(H_{2}, A\right)\right] \sqsubseteq g_{\varphi}\left(H_{1}, A\right) \sqcap g_{\varphi}\left(H_{2}, A\right)$

(vii) $g_{\varphi}^{-1}\left[\left(U_{1}, B\right) \sqcup\left(U_{2}, B\right)\right]=g_{\varphi}^{-1}\left(U_{1}, B\right) \sqcup g_{\varphi}^{-1}\left(U_{2}, B\right)$

(viii) $g_{\varphi}^{-1}\left[\left(U_{1}, B\right) \sqcap\left(U_{2}, B\right)\right]=g_{\varphi}^{-1}\left(U_{1}, B\right) \sqcap g_{\varphi}^{-1}\left(U_{2}, B\right)$

Definition 8 (see [18]). The collection $\tau$ of soft sets over $X$ under a fixed set of parameters $A$ is called an infrasoft topology on $X$ if it is closed under finite soft intersection as well as it contains $\Phi$.

The triple $(X, \tau, A)$ is called an infrasoft topological space. The term given to each member of $\tau$ is called an infrasoft open set, and the relative complement each member of $\tau$ is called an infrasoft closed set.
Definition 9 (see [18]). Let $(H, A)$ be a subset of $(X, \tau, A)$.

(i) The intersection of all infrasoft closed subsets of $(X, \tau, A)$ which contains a soft set $(H, A)$ is called the infrasoft closure points of $(H, A)$. It is denoted by $\mathrm{Cl}(H, A)$.

(ii) The union of all infrasoft open subsets of $(X, \tau, A)$ which are contained in a soft set $(H, A)$ is called the infrasoft interior points of $(H, A)$. It is denoted by $\operatorname{Int}(H, A)$.

Definition 10 (see [18]). Let $(X, \tau, A)$ be an infrasoft topological space and $U$ be a nonempty subset of $X$. A class $\tau_{U}=\{\widetilde{U} \sqcap(G, A):(G, A) \in \tau\}$ is called an infrasoft relative topology on $U$, and $\left(U, \vartheta_{U}, A\right)$ is called an infrasoft subspace of $(X, \tau, A)$.

Proposition 2 (see [18]). Suppose that $\Omega=\left\{\Omega_{a}\right\}_{a \in A}$ is a family of (crisp) classical infratopologies on $X$. Then,

$\tau(\boldsymbol{\Omega})=\left\{\{(a, H(a)): a \in A\} \in S\left(X_{A}\right)\right.$ such that $H(a) \in \Omega_{a}$ for each $\left.a \in A\right\}$,

which defines an infrasoft topology on $X$ (we called this type of infrasoft topology as an infrasoft topology generated by (crisp) classical infratopologies).

Theorem 1 (see [18]). A soft mapping $g_{\varphi}:(X, \tau, A) \longrightarrow(Y, \theta, B)$ is infrasoft continuous if and only if the inverse image of each infrasoft open (resp. infra soft closed) set is an infrasoft open (resp. infra soft closed) set.

Definition 11 (see [28]). Let $g: X \longrightarrow Y$ be a mapping. A subfamily $\theta$ of the power set $P(Y)$ of $Y$ is said to be a quotient topology over $Y$ (with respect to $g$ ) if $\theta$ is the largest topology that makes $g$ continuous.

\section{Note on Soft Mappings}

In this section, we target to achieve two goals; first, we update Definition 5 of soft mappings to be convenient for studying the concepts of open, closed, and homeomorphism mappings in different soft structures such as soft topology, suprasoft topology, and infrasoft topology. Second, we simplify the formulation of Definition 5 using a soft point as the starting point.

We begin by the following example which helps us to clarify the followed approach to achieve the coveted goals.

Example 1. Let $X=\{u, v, w\}$ and $Y=\{x, y, z\}$ be two universal sets, and let $A=\left\{a_{1}, a_{2}, a_{3}\right\}$ and $B=\left\{b_{1}, b_{2}, b_{3}\right\}$ be two sets of parameters. Consider a soft mapping $g_{\varphi}$ from $S\left(X_{A}\right)$ to $S\left(Y_{B}\right)$, where the mappings $g: X \longrightarrow Y$ and $\varphi: A \longrightarrow B$ are defined as follows:

$$
\begin{aligned}
g(u) & =g(v)=x, \\
g(w) & =z, \\
\varphi\left(a_{1}\right) & =\varphi\left(a_{2}\right)=b_{2}, \\
\varphi\left(a_{3}\right) & =b_{3} .
\end{aligned}
$$

For $\left.(H, A)=\left\{\left(a_{1},\{u\}\right),\left(a_{2}, X\right),\left(a_{3},\{w\}\right)\right\}\right\}$, we have $g_{\varphi}(H, A)=\left\{\left(b_{2},\{x, z\}\right),\left(b_{3},\{z\}\right)\right\}$. Note that, for any infrasoft topology (or any soft structure) on $X$ and $Y$ with the sets of parameters $A$ and $B$, a soft mapping $g_{\varphi}$ will not be soft open (soft closed) because the image of any soft sets under $g_{\varphi}$ is a soft subset of a soft set $\left\{\left(b_{2},\{x, z\}\right),\left(b_{3},\{x, z\}\right)\right\}$. To remove this shortcoming, we make a slight modification for Definition 5 to be appropriate for defining soft open and closed mappings.

Definition 12. The image of $(H, M) \in S\left(X_{A}\right)$ under a soft mapping $g_{\varphi}: S\left(X_{A}\right) \longrightarrow S\left(Y_{B}\right)$, where $g: X \longrightarrow Y$ and $\varphi: A \longrightarrow B$ are given by $g_{\varphi}(H, M)=(g(H), B)$ such that

$$
g(H)(b)= \begin{cases}\underset{a \in \varphi^{-1}(b) \cap M}{\cup} g(H(a)), & \varphi^{-1}(b) \neq \varnothing, \\ \varnothing, & \varphi^{-1}(b)=\varnothing,\end{cases}
$$

for each $b \in B$.

If there is no confusion, we simplify the above formulation as follows:

$$
g(H)(b)=g\left(\underset{a \in \varphi^{-1}(b) \cap M}{\cup} H(a)\right), \quad \text { for each } b \in B .
$$

The following result is easy, but it will be useful for the investigation. 


\section{Proposition 3}

(i) The image of each soft point is a soft point

(ii) The product of two soft points is a soft point

Definition 13. A relation $\Omega$ on $\widetilde{X}$ is a subset of $P\left(X_{A}\right) \times P\left(X_{A}\right)$.

Note that Definition 5 does not give meaning of a soft mapping as a self-contained concept. It only gives the method of calculating the image and preimage of soft sets. So, it is nature to wonder what is the formulation of soft mappings that simulates its counterpart on the (crisp) set theory? It is well known that a soft point represents the soft version of an ordinary point so that we redefine a soft mapping between two classes of soft points as follows.

Definition 14. Let $g: X \longrightarrow Y$ and $\varphi: A \longrightarrow B$ be two crisp mappings. A soft mapping $g_{\varphi}$ of $P\left(X_{A}\right)$ into $P\left(Y_{B}\right)$ is a relation such that each soft point in $P\left(X_{A}\right)$ is related to one and only one soft point in $P\left(Y_{B}\right)$ such that

$$
g_{\varphi}\left(P_{a}^{x}\right)=P_{\varphi(a)}^{g(x)}, \quad \text { for each } P_{a}^{x} \in P\left(X_{A}\right) .
$$

In addition, $g_{\varphi}^{-1}\left(P_{b}^{y}\right)=\sqcup_{\substack{a \in \varphi^{-1} \\ x \in g^{-1}(y)}} P_{a}^{x}$ for each $P_{b}^{y} \in P$
$\left(Y_{B}\right)$.

From the above definition, we note two matters; first, reduce calculation burden and its difficulty that arises from Definition 5. Second, Definition 14 gives a logical explanation (justification) for some soft concepts, for example, it can be easily seen why we determine that $g_{\varphi}$ is injective or surjective according to its two crisp mappings $g$ and $\varphi$.

Now, we prove the following results.

Lemma 1. Let $g_{\varphi}: P\left(X_{A}\right) \underset{P^{g(x)}}{\longrightarrow} P\left(Y_{B}\right)$ be a soft mapping. Then, $g_{\varphi}(H, A)=\sqcup_{P_{a}^{x} \in(H, A)} P_{\varphi(a)}^{g(x)}$.

Proof. Since it can be written a soft set as a soft union of its soft points, we obtain $g_{\varphi}(H, A)=g_{\varphi}\left(\sqcup_{P_{a}^{x} \epsilon}\right.$ $\left.(H, A) P_{a}^{x}\right)=\sqcup_{P_{a}^{x} \in(H, A)} g_{\varphi}\left(P_{a}^{x}\right)=\sqcup_{P_{a}^{x} \in(H, A)} P_{\varphi(a)}^{g(x)}, \quad$ as required.

Theorem 2. The image of soft sets obtained from Definitions 12 and 14 is identical, i.e., Definitions 12 and 14 are identical.

Proof. Let $(H, M)$ be a soft set in $S\left(X_{A}\right)$. Then, $g_{\varphi}(H, A)=g_{\varphi}\left(\sqcup_{P_{a}^{x} \in(H, A)} P_{a}^{x}\right)=\sqcup_{P_{a}^{x} \in(H, A)} g_{\varphi}\left(P_{a}^{x}\right)$. It follows from (i) of Proposition 3 that $g_{\varphi}\left(P_{a}^{x}\right)$ is a soft point in $S\left(Y_{B}\right)$. According to Definition 12, we obtain $g_{\varphi}\left(P_{a}^{x}\right)=P_{\varphi(a)}^{g(x)}$. Thus, $g_{\varphi}(H, M)=\sqcup_{P_{a}^{x} \in(H, A)} P_{\varphi(a)}^{g(x)}$ which represents the image of $(H, M)$ according to Definition 14 . Hence, we obtain the coveted result.

Corollary 1. The preimage of soft sets obtained from Definitions 6 and 14 is identical.

We complete this part by presenting some amendments of some results given in [18]. First, the following result is the correct form of Proposition 7 in [18].
Proposition 4. Let $(H, A)$ and $(F, A)$ be subsets of $(X, \tau, A)$, where $X$ and $A$ are finite sets. If $\operatorname{cl}(H, A) \sqcap \operatorname{cl}(F, A)=\Phi$, then $\operatorname{int}((H, A) \sqcup(F, A))=\operatorname{int}(H, A) \sqcup \operatorname{int}(F, A)$.

Proof. It is clear that $\operatorname{int}(H, A) \sqcup \operatorname{int}(F, A)$ int $((H, A)$ $\sqcup(F, A))$. Conversely, let $P_{a}^{x} \in \operatorname{int}((H, A) \sqcup(F, A))$. Then, $\tau$ contains an infrasoft open set $(G, A)$ such that $P_{a}^{x} \in(G, A) \sqsubseteq(G, A) \sqcup(F, A)$. Since $X$ and $A$ are finite sets, we consider $(G, A)$ as a smallest infrasoft open set containing $P_{a}^{x}$. Now, we have three cases:

Case 1: $(G, A) \sqsubseteq(G, A)$. Then, $P_{a}^{x} \in \operatorname{int}(G, A)$.

Case 2: $(G, A) \sqsubseteq(F, A)$. Then, $P_{a}^{x} \in \operatorname{int}(F, A)$.

Case 3: $(G, A) \sqsubseteq(G, A)$ and $(G, A) \sqsubseteq(F, A)$. Then, $(G, A) \sqcap(G, A) \neq \Phi$ and $(G, A) \sqcap(F, A) \neq \Phi$. Since $(G, A)$ is the smallest infrasoft open set containing $P_{a}^{x}$, $P_{a}^{x} \in \operatorname{cl}(G, A)$ and $P_{a}^{x} \in \operatorname{cl}(F, A)$. But this contradicts assumption $\mathrm{cl}(G, A) \sqcap \mathrm{cl}(F, A)=\Phi$. Therefore, the only valid cases are Case 1 and Case 2. Thus, $\operatorname{int}((G, A) \sqcup(F, A)) \sqsubseteq \operatorname{int}(G, A) \sqcup \operatorname{int}(F, A)$. Hence, the proof is complete.

Second, we replace Definition 24 by Theorem 8 (which are given in [18]) to keep the systematic line of defining infrasoft continuity, infrasoft openness, and infrasoft closedness. That is, we reformulate Definition 24 of an infrasoft continuous map as follows.

Definition 15. A soft mapping $g_{\varphi}:(X, \tau, A) \longrightarrow(Y, \theta, B)$ is said to be infrasoft continuous if the preimage of each infrasoft open set is infrasoft open.

\section{Infrasoft Homeomorphism Mappings}

In this section, we initiate the concepts of infrasoft open, infrasoft closed, and infrasoft homeomorphism mappings. We show the relationships among them and study some properties. We construct some counterexamples to explain some invalid results.

Definition 16. A soft mapping $g_{\varphi}:(X, \tau, A) \longrightarrow(Y, \theta, B)$ is said to be infrasoft open (resp. infra soft closed) if the image of each infrasoft open (resp. infra soft closed) set is an infrasoft open (resp. infra soft closed) set.

Proposition 5. If $g_{\varphi}:(X, \tau, A) \longrightarrow(Y, \theta, B)$ is an infrasoft open mapping, then the following statements hold.

$$
\begin{aligned}
& \text { (i) } g_{\varphi}(\operatorname{int}(H, A)) \sqsubseteq \operatorname{int}\left(g_{\varphi}(H, A)\right) \quad \text { for } \quad \text { each } \\
&(H, A) \in S(X)_{A} \\
& \text { (ii) } g_{\varphi}^{-1}(c l(F, B)) \sqsubseteq c l\left(g_{\varphi}^{-1}(F, B)\right) \text { for each }(F, B) \in S(Y)_{B}
\end{aligned}
$$

Proof. To prove (i), let $P_{b}^{y} \in g_{\varphi}(\operatorname{int}(H, A))$. Then, there exists a soft point $P_{a}^{x} \in \operatorname{int}(H, A)$ such that $g_{\varphi}\left(P_{a}^{x}\right)=P_{b}^{y}$. Therefore, there exists an infrasoft open set $(U, A)$ such that $P_{a}^{x} \in(U, A) \sqsubseteq(H, A)$. Obviously, $P_{b}^{y}=g_{\varphi}\left(P_{a}^{x}\right) \in g_{\varphi}(U, A) \sqsubseteq$ $g_{\varphi}(H, A)$. By hypothesis, $g_{\varphi}(U, A)$ is an infrasoft open set; hence, $g_{\varphi}(U, A) \sqsubseteq \operatorname{int}\left(g_{\varphi}(H, A)\right)$, as required.

One can prove (ii) using a similar technique. 
Corollary 2. If $g_{\varphi}:(X, \tau, A) \longrightarrow(Y, \theta, B)$ is an infrasoft open mapping, then the image of an infrasoft neighborhood of $P_{a}^{x} \in \widetilde{X}$ is an infrasoft neighborhood of $g_{\varphi}\left(P_{a}^{x}\right)$.

Proposition 6. If $g_{\varphi}:(X, \tau, A) \longrightarrow(Y, \theta, B)$ is an infrasoft closed mapping, then $c l\left(g_{\varphi}(H, A)\right) \sqsubseteq g_{\varphi}(c l(H, A))$ for each $(H, A) \in S(X)_{A}$.

Proof. Suppose that $P_{b}^{y} \notin g_{\varphi}(\mathrm{cl}(H, A))$. Then, $g_{\varphi}^{-1}\left(P_{b}^{y}\right)$ 드 $\operatorname{cl}(H, A)$. This means for each $P_{a}^{x} \in g_{\varphi}^{-1}\left(P_{b}^{y}\right)$, there exists an infrasoft open set $(U, A)$ containing $P_{a}^{x}$ such that $(U, A) \sqcap(H, A)=\Phi$. Therefore, $(U, A) \sqcap \mathrm{cl}(H, A)=\Phi$. Now, $g_{\varphi}(H, A) \sqsubseteq g_{\varphi}(\mathrm{cl}(H, A)) \sqsubseteq g_{\varphi}\left(U^{c}, A\right)$. By assumption, $P_{b}^{y}$ $\notin g_{\varphi}(\mathrm{cl}(H, A))$ so that $g_{\varphi}\left(P_{a}^{x}\right)=P_{b}^{y} \notin g_{\varphi}\left(U^{c}, A\right)$. Since $g_{\varphi}$ is an infrasoft closed mapping, then $\operatorname{cl}\left(g_{\varphi}(H, A)\right)$ 드 $g_{\varphi}\left(U^{c}, A\right)$. Thus, $P_{b}^{y} \notin \operatorname{cl}\left(g_{\varphi}(H, A)\right)$, as required.

In fact, some characterizations of soft open and closed mappings, which are the counterparts of infrasoft open and infrasoft closed mappings, are losing via the structure of infrasoft topology. The following example illustrates this observation.

Example 2. Consider the following soft sets over $X=\left\{w_{1}, w_{2}\right\}$ under a parameter set $A=\left\{a_{1}, a_{2}\right\}$ defined as follows:

$$
\begin{aligned}
& \left(U_{1}, A\right)=\left\{\left(a_{1},\left\{w_{1}\right\}\right),\left(a_{2},\left\{w_{1}\right\}\right)\right\}, \\
& \left(U_{2}, A\right)=\left\{\left(a_{1}, \varnothing\right),\left(a_{2},\left\{w_{2}\right\}\right)\right\} .
\end{aligned}
$$

Then, $\tau=\left\{\Phi, \widetilde{X},\left(U_{i}, A\right): i=1,2\right\}$ is an infrasoft topology on $X$. Consider $\theta$ is the indiscrete soft topology (of course, it will be an infrasoft topology) on $X$. Let a soft mapping $g_{\varphi}:(X, \theta, A) \longrightarrow(X, \tau, A)$ be defined as follows:

$$
\begin{aligned}
& g_{\varphi}\left(P_{a_{1}}^{w_{1}}\right)=g_{\varphi}\left(P_{a_{1}}^{w_{2}}\right)=P_{a_{1}}^{w_{1}}, \\
& g_{\varphi}\left(P_{a_{2}}^{w_{1}}\right)=P_{a_{2}}^{w_{1}}, \\
& g_{\varphi}\left(P_{a_{2}}^{w_{2}}\right)=P_{a_{2}}^{w_{2}} .
\end{aligned}
$$

One can check that the two conditions given in Proposition 5 hold. Also, the image of any infrasoft neighborhood of $P_{a}^{w}$ is an infrasoft neighborhood of $g_{\varphi}\left(P_{a}^{w}\right)$. Moreover, $\operatorname{cl}\left(g_{\varphi}(H, A)\right) \sqsubseteq g_{\varphi}(\operatorname{cl}(H, A))$ for each $(H, A) \in S(X)_{A}$. On the contrary, the image of an infrasoft clopen set $\widetilde{X}$ is $\left\{\left(a_{1},\left\{w_{1}\right\}\right),\left(a_{2}, X\right)\right\}$ which is neither infrasoft open nor infrasoft closed in $\tau$; hence, $g_{\varphi}$ is neither an infrasoft open mapping nor an infrasoft closed mapping.

Proposition 7. Let $g_{\varphi}:(X, \tau, A) \longrightarrow(Y, \theta, B)$ be a bijective soft map. Then, $g_{\varphi}$ is infrasoft open if and only if it is infrasoft closed.

Proof. The proof follows from the fact that a bijective soft map $g_{\varphi}$ implies that $g_{\varphi}\left(H^{c}, A\right)=\left(g_{\varphi}(H, A)\right)^{c}$.

Proposition 8. Let $g_{\varphi}:(X, \tau, A) \longrightarrow(Y, \theta, B)$ be an infrasoft open mapping and $\widetilde{U}$ be an infrasoft open set in $\tau$. Then, $g_{\varphi \mid U}:\left(U, \tau_{U}, A\right) \longrightarrow(Y, \theta, B)$ is an infrasoft open mapping.
Proof. Let $(G, A)$ be an infrasoft open set in $\tau_{U}$. Then, there is an infrasoft open set $(V, A)$ in $\tau$ such that $(G, A)=(V, A) \sqcap \widetilde{U}$. This means that $(G, A)$ is also an infrasoft open set in $\tau$. By hypothesis, $g_{\varphi \mid U}(G, A)=g_{\varphi}(G, A)$ is an infrasoft open set in $\theta$; hence, $g_{\varphi \mid U}$ is an infrasoft open mapping.

Definition 17. The composition of two soft mappings $f_{\psi}:(X, \tau, A) \longrightarrow(Y, \theta, B)$ and $g_{\varphi}:(Y, \theta, B) \longrightarrow(Z, v, C)$ is a soft mapping $g_{\varphi} \circ f_{\psi}:(X, \tau, A) \longrightarrow(Z, v, C)$ such that $\left(g_{\varphi} \circ f_{\psi}\right)\left(P_{a}^{x}\right)=g_{\varphi}\left(f_{\psi}\left(P_{a}^{x}\right)\right)$.

Proposition 9. Let $g_{\varphi}:(X, \tau, A) \longrightarrow(Y, \theta, B)$ and $f_{\psi}:(Y, \theta, B) \longrightarrow(Z, \mu, C)$ be two infrasoft mappings. Then, the following statements hold.

(i) If $g_{\varphi}$ and $f_{\psi}$ are infrasoft open mappings, then $f_{\psi} \circ g_{\varphi}$ is an infrasoft open mapping

(ii) If $f_{\psi} \circ g_{\varphi}$ is an infrasoft open mapping and $g_{\varphi}$ is a surjective infrasoft continuous mapping, then $f_{\psi}$ is an infrasoft open mapping

(iii) If $f_{\psi} \circ g_{\varphi}$ is an infrasoft open mapping and $f_{\psi}$ is an injective infrasoft continuous mapping, then $g_{\varphi}$ is an infrasoft open mapping

Proof

(i) Straightforward.

(ii) Let $(G, B)$ be an infrasoft open set in $\theta$. By hypothesis, $g_{\varphi}^{-1}(G, B)$ is an infrasoft open set in $\tau$; therefore, $\left(f_{\psi} \circ g_{\varphi}\right)\left(g_{\varphi}^{-1}(G, B)\right)$ is an infrasoft open set in $\mu$. Since $g_{\varphi}$ is surjective, then $\left(f_{\psi} \circ g_{\varphi}\right)\left(g_{\varphi}^{-1}(G, B)\right)=f_{\psi}\left(g_{\varphi}\left(g_{\varphi}^{-1}(G, B)\right)\right)=f_{\psi}$ $(G, B)$. Thus, $f_{\psi}$ is an infrasoft open mapping.

(iii) Let $(G, A)$ be an infrasoft open set in $\tau$. By hypothesis, $\left(f_{\psi} \circ g_{\varphi}\right)(G, A)$ is an infrasoft open set in $\mu$; therefore, $f_{\psi}^{-1}\left(f_{\psi} \circ g_{\varphi}(G, A)\right)$ is an infrasoft open set in $\theta$. Since $f_{\psi}$ is injective, then $f_{\psi}^{-1}\left(f_{\psi} \circ g_{\varphi}\right.$ $(G, A))=\left(f_{\psi}^{-1} f_{\psi}\right) \quad\left(g_{\varphi}(G, A)\right)=g_{\varphi}(G, A)$. Thus, $g_{\varphi}$ is an infrasoft open mapping.

Following similar arguments given in the above proof, one can prove the next result.

Proposition 10. Let $g_{\varphi}:(X, \tau, A) \longrightarrow(Y, \theta, B)$ and $f_{\psi}:(Y, \theta, B) \longrightarrow(Z, \mu, C)$ be two infrasoft mappings. Then, the following statements hold.

(i) If $g_{\varphi}$ and $f_{\psi}$ are infrasoft closed mappings, then $f_{\psi} \circ g_{\varphi}$ is an infrasoft closed mapping

(ii) If $f_{\psi} \circ g_{\varphi}$ is an infrasoft closed mapping and $g_{\varphi}$ is a surjective infrasoft continuous mapping, then $f_{\psi}$ is an infrasoft closed mapping

(iii) If $f_{\psi} \circ g_{\varphi}$ is an infrasoft closed mapping and $f_{\psi}$ is an injective infrasoft continuous mapping, then $g_{\varphi}$ is an infrasoft closed mapping 
Proposition 11. Let $\left\{\left(X_{i}, \tau_{i}, A_{i}\right): i \in I\right\}$ be a family of infrasoft topological spaces. Then, $\tau=\left\{\prod_{i \in I}\left(U_{i}, A_{i}\right)\right.$ : $\left.\left(U_{i}, A_{i}\right) \in \tau_{i}\right\}$ is an infrasoft topology on $\mathbf{X}=\prod_{i \in I} X_{i}$ under a set of parameters $\mathbf{A}=\prod_{i \in I} A_{i}$.

Proof. It is clear that $\widetilde{\mathbf{X}}$ and $\Phi$ are members in $\tau$. Now, let $\prod_{i \in I}\left(U_{i}, A_{i}\right)$ and $\prod_{i \in I}\left(V_{i}, A_{i}\right)$ are two members in $\tau$. Since $\left(U_{i}, A_{i}\right) \sqcap\left(V_{i}, A_{i}\right) \in \tau_{i}$ for each $i$, then it follows from the fact $\left(\prod_{i \in I}\left(U_{i}, A_{i}\right)\right) \sqcap\left(\prod_{i \in I}\left(V_{i}, A_{i}\right)\right)=\prod_{i \in I}\left(\left(U_{i}, A_{i}\right) \sqcap\left(V_{i}, A_{i}\right)\right)$ that $\tau$ is closed under finite soft intersection. Hence, the proof is complete.

We call $\tau$ given in the proposition above a product of infra soft topologies, and $(\mathbf{X}, \tau, \mathbf{A})$ a product of infrasoft spaces.

Definition 18. Let $\left\{g_{i \varphi}:\left(X_{i}, \tau_{i}, A_{i}\right) \longrightarrow\left(Y_{i}, \theta_{i}, B_{i}\right): i \in I\right\}$ be a family of soft mappings. The product of these soft mappings is given by $g_{\varphi}:(\mathbf{X}, \tau, \mathbf{A}) \longrightarrow(\mathbf{Y}, \theta, \mathbf{B})$ such that $g_{\varphi}\left(P_{\left(a_{i}\right)_{i \in I}}^{\left(x_{i}\right)_{i \in I}}\right)=\left(g_{\varphi}\left(P_{a_{i}}^{x_{i}}\right)\right)_{i \in I}$

If $I$ is countable, then we write it as follows:

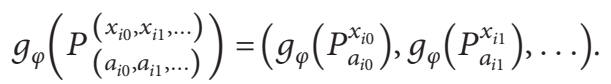

Theorem 3. The product of infrasoft open mappings is an infrasoft open mapping.

Proof. Let $g_{\varphi}:(\mathbf{X}, \tau, \mathbf{A}) \longrightarrow(\mathbf{Y}, \theta, \mathbf{B})$ be the product of infrasoft open mappings of the family $\left\{g_{i \varphi}:\left(X_{i}, \tau_{i}, A_{i}\right)\right.$ $\left.\longrightarrow\left(Y_{i}, \theta_{i}, B_{i}\right): i \in I\right\}$. Let $(U, \mathbf{A})$ be an infrasoft open set in $\tau$. Since $(U, \mathbf{A})=\prod_{i \in I}\left(U_{i}, A_{i}\right)$ where $\left(U_{i}, A_{i}\right) \in \tau_{i}$ for each $i$, then $g_{\varphi}(U, \mathbf{A})=g_{\varphi}\left(\prod_{i \in I}\left(U_{i}, A_{i}\right)\right)=\prod_{i \in I} g_{i \varphi}\left(U_{i}, A_{i}\right)$. By hypothesis, $g_{i \varphi}\left(U_{i}, A_{i}\right)$ is an infrasoft open set in $\theta_{i}$ for each $i$. According to the definition of the product of infrasoft spaces, $g_{\varphi}(U, \mathbf{A})$ is an infrasoft open set in $\theta$. Hence, we obtain the coveted result.

Corollary 3. The product of infrasoft closed mappings $\left\{g_{i \varphi}: i \in I\right\}$ is an infrasoft closed mapping provided that $g_{i \varphi}$ is bijective for each $i$.

Theorem 4. The product of infrasoft continuous mappings is an infrasoft continuous mapping.

Proof. Similar to the proof of Theorem 3, one can prove this result.

Theorem 5. If $g_{\varphi}:(X, \tau, A) \longrightarrow(Y, \theta, B)$ is an infrasoft open (resp. infrasoft closed) mapping such that $\varphi$ is injective, then $g:\left(X, \tau_{a}\right) \longrightarrow\left(Y, \theta_{\varphi(a)}\right)$ is an infraopen (resp. infraclosed) mapping.

Proof. Let $U$ be an infraopen set in $\tau_{a}$. Then, there exists an infrasoft open set $(H, A)$ in $\tau$ such that $H(a)=U$. Since $g_{\varphi}$ is an infrasoft open mapping, then $g_{\varphi}(H, A)=(g(H), B)$ is an infrasoft open set in $\theta$. Therefore, $g(H)(b=\varphi(a))=$ $g\left(\cup_{a \in \varphi^{-1}(b)} H(a)\right)$ is infrasoft open in $\theta_{\varphi(a)}$. Since $\varphi$ is injective, then $g(H)(b=\varphi(a))=g\left(\cup_{a=\varphi^{-1}(b)} H(a)\right)=g(U)$.
Thus, $g(U)$ is an infraopen set in $\theta_{\varphi(a)}$. Hence, we obtain the coveted result.

Following the same arguments, the case between parenthesis can be proved.

Example below explains that the converse of the above theorem fails.

Example 3. Let $A=\left\{a_{1}, a_{2}\right\}$, and consider the two infrasoft topologies $\tau=\{\Phi, \widetilde{X},(U, A)\}$ and $\theta=\{\Phi, \widetilde{X},(V, A)\}$ on $X=\left\{w_{1}, w_{2}\right\}$, where

$$
\begin{aligned}
& (U, A)=\left\{\left(a_{1},\left\{w_{1}\right\}\right),\left(a_{2}, \varnothing\right)\right\}, \\
& (V, A)=\left\{\left(a_{1}, \varnothing\right),\left(a_{2},\left\{w_{1}\right\}\right)\right\} .
\end{aligned}
$$

Taking $g_{\varphi}:(X, \tau, A) \longrightarrow(X, \theta, A)$ as the soft identity mapping, it is clear that $g:\left(X, \tau_{a}\right) \longrightarrow\left(X, \tau_{\varphi(a)=a}\right)$ is an infraopen mapping and an infraclosed mapping for each $a \in A$. But $g_{\varphi}$ is neither an infrasoft open mapping nor an infrasoft closed mapping because $g_{\varphi}(U, A)=(U, A)$ is not an infrasoft open set in $\theta$ and $g_{\varphi}\left(U^{c}, A\right)=\left(U^{c}, A\right)$ is not an infrasoft closed set in $\theta$.

We show under which condition the converse of Theorem 5 is true.

Theorem 6. Let $\theta$ be an infrasoft topology induced from the (crisp) classical infratopologies and $g_{\varphi}:(X, \tau, A) \longrightarrow$ $(Y, \theta, B)$ be a soft mapping such that $\varphi$ is injective. Then, $g_{\varphi}:(X, \tau, A) \longrightarrow(Y, \theta, B)$ is infrasoft open (resp. infrasoft closed) if and only if $g:\left(X, \tau_{a}\right) \longrightarrow\left(Y, \theta_{\varphi(a)}\right)$ is infraopen (resp. infraclosed).

Proof. Necessity: it follows from Theorem 5.

Sufficiency: let $(H, A)$ be an infrasoft open set in $\tau$. Since $g:\left(X, \tau_{a}\right) \longrightarrow\left(Y, \theta_{\varphi(a)}\right)$ is an infraopen mapping, then $g(H(a))$ is an infraopen set in $\theta_{\varphi(a)}$ for each $a \in A$. Now, $g_{\varphi}(H, A)=(g(H), B)$ such that either $g(H)(b=\varphi(a))=$ $g\left(\cup_{a=\varphi^{-1}(b)} H(a)\right)=g(H(a))$ is an infraopen set in $\theta_{\varphi(a)}$ or $g(H)(b)=g\left(\cup_{\varphi^{-1}(b)=\varnothing} H(a)\right)=\varnothing$ is an infraopen set in $\theta_{b}$. Since $\theta$ is generated from the crisp infratopologies, then $g_{\varphi}(H, A)$ is an infrasoft open set in $\theta$. Hence, the proof is complete.

Following the same arguments, the case between parenthesis can be proved.

Definition 19. A bijective soft mapping $g_{\varphi}:(X, \tau, A)$ $\longrightarrow(Y, \theta, B)$ is said to be an infrasoft homeomorphism if it is infrasoft continuous and infrasoft open.

We cancel the proofs of the next two propositions because they are easy.

Proposition 12. Let $g_{\varphi}:(X, \tau, A) \longrightarrow(Y, \theta, B)$ and $f_{\psi}:(Y, \theta, B) \longrightarrow(Z, \mu, C)$ be two infrasoft homeomorphism mappings. Then, $f_{\psi} \circ g_{\varphi}$ is an infrasoft homeomorphism mapping.

Proposition 13. If $g_{\varphi}:(X, \tau, A) \longrightarrow(Y, \theta, B)$ is a bijective soft mapping, then the following statements are equivalent.

(i) $g_{\varphi}$ is an infrasoft homeomorphism 
(ii) $g_{\varphi}$ and $g_{\varphi}^{-1}$ are infrasoft continuous

(iii) $g_{\varphi}$ is infrasoft closed and infrasoft continuous

The proofs of the following two theorems follow from Theorems 5 and 6, respectively.

Theorem 7. If a soft mapping $f_{\varphi}:(X, \tau, A) \longrightarrow(Y, \theta, B)$ is infrasoft homeomorphism, then a mapping $f:\left(X, \tau_{a}\right)$ $\longrightarrow\left(Y, \theta_{\varphi(a)}\right)$ is infrahomeomorphism.

Theorem 8. Let $\theta$ be an infrasoft topology induced from classical (crisp) infratopologies. Then, a soft mapping $f_{\varphi}:(X, \tau, A) \longrightarrow(Y, \theta, B)$ is infrasoft homeomorphism if and only if a mapping $f:\left(X, \tau_{a}\right) \longrightarrow\left(Y, \theta_{\varphi(a)}\right)$ is infrahomeomorphism.

Definition 20. A soft subset $(H, A)$ of an infrasoft topological space $(X, \tau, A)$ is called an isolated soft set if there exists a soft point $P_{a}^{x} \in(H, A)$ such that $P_{a}^{x} \notin(H, A)^{i}$.

Proposition 14. If $g_{\varphi}:(X, \tau, A) \longrightarrow(Y, \theta, B)$ is an infrasoft homeomorphism mapping, then the following statements hold for each $(H, A) \in S(X)_{A}$.

(i) $g_{\varphi}(\operatorname{int}(H, A))=\operatorname{int}\left(g_{\varphi}(H, A)\right)$

(ii) $g_{\varphi}(c l(H, A))=c l\left(g_{\varphi}(H, A)\right)$

(iii) $g_{\varphi}\left((H, A)^{i \prime}\right)=\left(g_{\varphi}(H, A)\right)^{i,}$

Proof. We prove (i), and one can prove the other two cases similarly.

It follows from (i) of Proposition 5 that $g_{\varphi}(\operatorname{int}(H, A)) \sqsubseteq \operatorname{int}\left(g_{\varphi}(H, A)\right)$. Conversely, let $P_{b}^{y} \in \operatorname{int}\left(g_{\varphi}\right.$ $(H, A)$. Then, there exists an infrasoft open set $(U, B)$ such that $P_{b}^{y} \in(U, B) \sqsubseteq g_{\varphi}(H, A)$. By hypothesis, $P_{a}^{x}=g_{\varphi}^{-1}$ $\left(P_{b}^{y}\right) \in g_{\varphi}^{-1}(U, B) \sqsubseteq(H, A)$ such that $g_{\varphi}^{-1}(U, B)$ is an infrasoft open set in $\tau$. Therefore, $P_{a}^{x} \in \operatorname{int}(H, A)$. This means that $P_{b}^{y} \in g_{\varphi}(\operatorname{int}(H, A))$, as required.

Definition 21. A property is said to be an infrasoft topological invariant if the property possessed by an infratopological space $(X, \tau, A)$ is also possessed by each an infrasoft homeomorphic to $(X, \tau, A)$.

Theorem 9. The property of an infrasoft dense set (isolated soft set) is an infrasoft topological invariant.

Proof. Let $g_{\varphi}:(X, \tau, A) \longrightarrow(Y, \theta, B)$ be an infrasoft homeomorphism mapping, and let $(H, A)$ an infrasoft dense subset of $(X, \tau, A)$, i.e., $\mathrm{cl}(H, A)=\widetilde{X}$. It follows from (ii) of Proposition 14 that $\operatorname{cl}\left(g_{\varphi}(H, A)\right)=g_{\varphi}(\operatorname{cl}(H, A))=g_{\varphi}(\widetilde{X})$ $=\operatorname{cl}(\widetilde{Y})=\widetilde{Y}$. Therefore, $g_{\varphi}(H, A)$ is an infrasoft dense subset of $(Y, \theta, B)$. Hence, the proof is complete.

Theorem 10. The product of two infrasoft homeomorphism mappings is an infrasoft homeomorphism mapping.
Proof. It is clear that the product of bijective soft mappings is bijective. Then, Theorem 3 and Corollary 4.15 finish the coveted result.

Proposition 15. Let $\left\{\left(X_{i}, \tau_{i}, A\right): i \in I\right\}$ be a family of pairwise disjoint infrasoft topological spaces. Then, the family $\tau=$ $\left\{(U, A):(U, A) \sqcap \widetilde{X}_{i}\right\}$ is an infrasoft open set in $\left(X_{i}, \tau_{i}, A\right)$ for each $i \in I\}$ produces an infrasoft topology on $X=\cup_{i \in I} X_{i}$ with a constant set of parameters $A$.

Proof. It is clear that $\widetilde{X}$ and $\Phi$ are members of $\tau$. To prove that $\tau$ is closed under finite soft intersections, let $\left(U_{1}, A\right)$ and $\left(U_{2}, A\right)$ be two members of $\tau$. Then, $\left(U_{1}, A\right) \sqcap \widetilde{X}_{i} \in \tau_{i}$ and $\left(U_{2}, A\right) \sqcap \widetilde{X}_{i} \in \tau_{i}$ for each $i \in I$. Therefore, $\left[\left(U_{1}, A\right) \sqcap\right.$ $\left.\left(U_{2}, A\right)\right] \sqcap \bar{X}_{i} \in \tau_{i}$ for each $i \in I$. Thus, $\left(U_{1}, A\right) \sqcap\left(U_{2}, A\right) \in \tau$. Hence, $\tau$ is an infrasoft topology on $X$.

We call the infrasoft topological space given in the above proposition a sum of infrasoft topological spaces and is denoted by $\left(\oplus X_{i}, \tau, A\right)$.

Proposition 16. A soft subset $(H, A)$ of $\left(\oplus X_{i}, \tau, A\right)$ is infrasoft closed if and only if $(H, A) \sqcap \widetilde{X}_{i}$ is an infrasoft closed set in $\tau_{i}$ for each $i \in I$.

Proof. $\quad(H, A)$ is an infra soft closed set in $\tau \Leftrightarrow\left(H^{c}, A\right) \sqcap \widetilde{X}_{i}$ is an infra soft open set in $\tau_{i}$ for any $i \in I \Leftrightarrow(H, A) \sqcap \widetilde{X}_{i}$ is an infra soft closed set in $\tau_{i}$ for any $i \in I$.

Definition 22. Let $\left\{g_{i \varphi}:\left(X_{i}, \tau_{i}, A\right) \longrightarrow\left(Y_{i}, \theta_{i}, B\right): i \in I\right\}$ be a family of soft mappings. Then, we define a soft mapping $g_{\varphi}:\left(\oplus X_{i}, \tau, A\right) \longrightarrow\left(\oplus Y_{i}, \theta, B\right)$ as follows: the image of each $(U, A) \sqsubseteq \oplus X_{i}$ and the image of each $(V, B) \sqsubseteq \oplus Y_{i}$ are given by

(1) $g_{\varphi}(U, A)=\sqcup_{i \in I} g_{i \varphi}\left(\left[(U, A) \sqcap \widetilde{X}_{i}\right]\right)$

(2) $g_{\varphi}^{-1}(V, B)=\sqcup_{i \in I} g_{i \varphi}^{-1}\left(\left[(V, B) \sqcap \widetilde{X}_{i}\right]\right)$

Theorem 11. A soft mapping $g_{\varphi}:\left(\oplus X_{i}, \tau, A\right) \longrightarrow\left(\oplus Y_{i}, \theta, B\right.$ ) is infrasoft open (resp. infrasoft closed) if and only if all soft mappings $g_{i \varphi}:\left(X_{i}, \tau_{i}, A\right) \longrightarrow\left(Y_{i}, \theta_{i}, B\right)$ are infrasoft open (resp. infrasoft closed).

Proof. We prove the theorem in case of the soft mapping is infra soft open.

Necessity: let $g_{\varphi}:\left(\oplus X_{i}, \tau, A\right) \longrightarrow\left(\oplus Y_{i}, \theta, B\right)$ be an infrasoft open mapping. Taking an arbitrary soft mapping $g_{j \varphi}:\left(X_{j}, \tau_{j}, A\right) \longrightarrow\left(Y_{j}, \theta_{j}, B\right)$, where $j \in I$, let $(U, A)$ be an infrasoft open set in $\tau_{j}$. Then, $(U, A)$ is an infrasoft open set in $\tau$. Therefore, $g_{\varphi}(U, A)$ is an infrasoft open set in $\theta$. Since $(U, A) \sqcap \widetilde{X}_{i}=\Phi$ for each $i \neq j$, then $g_{\varphi}(U, A)=g_{j \varphi}(U, A)$. Thus, $g_{j \varphi}(U, A)$ is an infrasoft open set in $\theta_{j}$, as required.

Sufficiency: let $g_{i \varphi}:\left(X_{i}, \tau_{i}, A\right) \longrightarrow\left(Y_{i}, \theta_{i}, B\right)$ be an infrasoft open mapping for each $i \in I$, and let $(U, A)$ be an infrasoft open set in $\tau$. Since $(U, A) \sqcap \widetilde{X}_{i}$ is an infrasoft open set in $\tau_{i}$ for each $i \in I$, then $g_{i \varphi}\left[(U, A) \sqcap \widetilde{X}_{i}\right]$ is an infrasoft open set in $\theta_{i}$ for each $i \in I$. According to the definition of sum of infrasoft topologies, we obtain $\sqcup_{i \in I} g_{i \varphi}\left[(U, A) \sqcap \widetilde{X}_{i}\right]$ is an infrasoft open set in $\theta$. Now, $g_{\varphi}(U, A)=\sqcup_{i \in I} g_{i \varphi}\left[(U, A) \sqcap \widetilde{X}_{i}\right]$ so that $g_{\varphi}(U, A)$ is an infrasoft open set in $\left(\oplus Y_{i}, \theta, A\right)$. 
Following similar arguments, one can prove the case between the parenthesis.

Corollary 4. A soft mapping $g_{\varphi}:\left(\oplus X_{i}, \tau, A\right) \longrightarrow\left(\oplus Y_{i}, \theta, B\right)$ is infrasoft homeomorphism if and only if all soft mappings $g_{i \varphi}:\left(X_{i}, \tau_{i}, A\right) \longrightarrow\left(Y_{i}, \theta_{i}, B\right)$ are infrasoft homeomorphism.

\section{Infrasoft Quotient Mappings}

In this section, we define the concepts of quotient infrasoft topologies and infrasoft quotient mappings. We establish their main properties and investigate transmission of them to (crisp) mappings defined between parametric infrasoft topological spaces.

Definition 23. Let $g_{\varphi}:(X, \tau, A) \longrightarrow P\left(Y_{B}\right)$ be a soft mapping. A family $\theta \sqsubseteq S\left(Y_{B}\right)$ is said to be a quotient infrasoft topology over $Y$ (with respect to $g_{\varphi}$ ) if $\theta$ is the largest infrasoft topology that makes $g_{\varphi}$ infrasoft continuous.

Note that $\theta=\left\{(U, B) \sqsubseteq \widetilde{Y}: \mathscr{g}_{\varphi}^{-1}(U, B) \in \tau\right\}$.

The following example points out how a quotient infrasoft topology is constructed.

Example 4. Let $\tau=\left\{\Phi, \widetilde{X},\left(H_{1}, A\right),\left(H_{2}, A\right)\right\}$ be an infrasoft topology on $X=\{u, v, w\}$ with a set of parameters $A=\left\{a_{1}, a_{2}\right\}$ such that

$$
\begin{aligned}
& \left(H_{1}, A\right)=\left\{\left(a_{1},\{u\}\right),\left(a_{2}, X\right)\right\}, \\
& \left(H_{2}, A\right)=\left\{\left(a_{1},\{v\}\right),\left(a_{2}, \varnothing\right)\right\} .
\end{aligned}
$$

Let $Y=\{x, y, z\}$ be another universal set with a set of parameters $B=\left\{b_{1}, b_{2}\right\}$, and consider a soft mapping $g_{\varphi}$ from $(X, \tau, A)$ to $P\left(Y_{B}\right)$, where $g: X \longrightarrow Y$ and $\varphi: A \longrightarrow B$ are defined as follows:

$$
\begin{aligned}
g(u) & =x, \\
g(v) & =g(w)=y, \\
\varphi\left(a_{1}\right) & =b_{1}, \\
\varphi\left(a_{2}\right) & =b_{2} .
\end{aligned}
$$

Then, $\theta=\left\{\Phi, \tilde{Y},\left(U_{i}, B\right): i=1,2, \ldots, 10\right\}$ is the quotient infrasoft topology on $Y$ (with respect to $g_{\varphi}$ ), where

$$
\begin{aligned}
& \left(U_{1}, B\right)=\left\{\left(b_{1},\{x\}\right),\left(b_{2},\{x, y\}\right)\right\}, \\
& \left(U_{2}, B\right)=\left\{\left(b_{1},\{x\}\right),\left(b_{2}, Y\right)\right\}, \\
& \left(U_{3}, B\right)=\left\{\left(b_{1},\{x, z\}\right),\left(b_{2},\{x, y\}\right)\right\}, \\
& \left(U_{4}, B\right)=\left\{\left(b_{1},\{x, z\}\right),\left(b_{2}, Y\right)\right\}, \\
& \left(U_{5}, B\right)=\left\{\left(b_{1},\{x, y\}\right),\left(b_{2},\{x, y\}\right)\right\}, \\
& \left(U_{6}, B\right)=\left\{\left(b_{1}, Y\right),\left(b_{2},\{x, y\}\right)\right\}, \\
& \left(U_{7}, B\right)=\left\{\left(b_{1},\{x, y\}\right),\left(b_{2}, Y\right)\right\}, \\
& \left(U_{8}, B\right)=\left\{\left(b_{1},\{z\}\right),\left(b_{2}, \varnothing\right)\right\}, \\
& \left(U_{9}, B\right)=\left\{\left(b_{1}, \varnothing\right),\left(b_{2},\{z\}\right)\right\}, \\
& \left(U_{10}, B\right)=\left\{\left(b_{1},\{z\}\right),\left(b_{2},\{z\}\right)\right\} .
\end{aligned}
$$

Note that, for any infrasoft topology on $Y$ is proper finer than $\theta, g_{\varphi}$ is not infrasoft continuous.

Theorem 12. Let $g_{\varphi}:(X, \tau, A) \longrightarrow(Y, \theta, B)$ be an infrasoft continuous mapping. Then, the following statements are equivalent.

(i) $\theta$ is a quotient infrasoft topology

(ii) $(U, B)$ is an infrasoft open set in $\theta$ if $g_{\varphi}^{-1}(U, B)$ is an infrasoft open set in $\tau$

(iii) $(V, B)$ is an infrasoft closed set if $g_{\varphi}^{-1}(V, B)$ is an infrasoft closed set

Proof

(i) $\Longrightarrow$ (ii): since $g_{\varphi}$ is infrasoft continuous, then $g_{\varphi}^{-1}(U, B)$ is an infrasoft open set in $\tau$ for each infrasoft open set $(U, B)$ in $\theta$. Conversely, let $g_{\varphi}^{-1}(U, B)$ be an infrasoft open set in $\tau$. Since $\theta$ is a quotient infrasoft topology, then $(U, B) \in \theta$, as required.

(ii) $\Longrightarrow$ (i): suppose that there exists an infrasoft topology $v$ that makes $g_{\varphi}$ infrasoft continuous such that $\theta \sqsubseteq \nu$. Now, let $(U, B) \in \nu$. Then, $g_{\varphi}^{-1}(U, B) \in \tau$. By hypothesis, $(U, B) \in \theta$. Thus, $\theta=v$, as required.

(ii) $\Longrightarrow$ (iii): straightforward.

Corollary 5. If $g_{\varphi}$ is an infrasoft continuous mapping from $(X, \tau, A)$ onto $(Y, \theta, B)$ such that $g_{\varphi}$ is either infrasoft open or infrasoft closed, then $\theta$ is a quotient infrasoft topology.

Proof. It is clear that $g_{\varphi}^{-1}(U, B)$ is an infrasoft open set in $\tau$ for each infrasoft open set $(U, B)$ in $\theta$ on one hand. On the other hand, $g_{\varphi}$ is a surjective; then, $(U, B)=g_{\varphi}\left(g_{\varphi}^{-1}(U, B)\right)$, and since $g_{\varphi}$ is an infrasoft open mapping, then $g_{\varphi}\left(g_{\varphi}^{-1}(U, B)\right)$ is an infrasoft open set so that $(U, B)$ is infrasoft open. It follows from (ii) of the above theorem that $\theta$ is a quotient infrasoft topology, as required.

The case of $g_{\varphi}$ is an infrasoft closed mapping is proved in a similar manner.

Proposition 17. Let $f_{\psi}$ be an infrasoft continuous mapping from $(X, \tau, A)$ onto a quotient infrasoft topological space $(Y, \theta, B)$. Then, $g_{\varphi}:(Y, \theta, B) \longrightarrow(Z, \mu, C)$ is infrasoft continuous if $g_{\varphi} \circ f_{\psi}$ is infrasoft continuous.

Proof. Necessity: it follows from the fact that the composition of two infrasoft continuous mappings is an infrasoft continuous mapping.

Sufficiency: suppose that $(U, C)$ is an infrasoft open set in $\mu$. Since $g_{\varphi} \circ f_{\psi}$ is infrasoft continuous, then $\left(g_{\varphi} \circ f_{\psi}\right)^{-1}(U, C)=f_{\psi}^{-1}\left(g_{\varphi}^{-1}(U, C)\right)$ is an infrasoft open set in $\tau$. Since $\theta$ is a quotient infrasoft topology, it follows from Theorem 12 that $g_{\varphi}^{-1}(U, C)$ is an infrasoft open set in $\theta$. Hence, we obtain the coveted result.

Recall that a mapping $g: X \longrightarrow Y$ is called a quotient inframapping if it is surjective and $Y$ is equipped with the quotient infratopology with respect to $g$. 
Definition 24. A soft mapping $g_{\varphi}: P\left(X_{A}\right) \longrightarrow P\left(Y_{B}\right)$ is said to be a quotient infrasoft mapping if $g_{\varphi}$ is surjective and $Y$ is equipped with the quotient infrasoft topology with respect to $g_{\varphi}$.

In other words, $g_{\varphi}: P\left(X_{A}\right) \longrightarrow P\left(Y_{B}\right)$ is said to be a quotient infrasoft mapping if $g_{\varphi}$ is surjective and a subset $(U, B)$ of $\tilde{Y}$ is infrasoft open if $g_{\varphi}^{-1}(U, B)$ is infrasoft open.

Proposition 18. If $g_{\varphi}:(X, \tau, A) \longrightarrow(Y, \theta, B)$ be a quotient infrasoft mapping such that $\tau$ generated from crisp infratopologies, then $g:\left(X, \tau_{a}\right) \longrightarrow\left(Y, \theta_{\varphi(a)}\right)$ is a quotient inframapping.

Proof. Firstly, it is clear that $g$ is a surjective mapping. To prove that $\theta_{\varphi(a)}$ is a quotient infratopology, let $U$ be an infraopen set in $\theta_{\varphi(a)}$. Then, there is an infrasoft open set $(H, B)$ in $\theta$ such that $H(\varphi(a))=U$. Therefore, $g_{\varphi}^{-1}(H, B)=$ $\left(g^{-1}(H), A\right)$ is an infrasoft open set in $\tau$; thus, $g^{-1}(H)(a)=$ $g^{-1}\left(H(\varphi(a))=g^{-1}(U)\right.$ is an infrasoft open set in $\tau_{a}$. Conversely, let $g^{-1}(U)$ be an infrasoft open set in $\tau_{a}$. Then, there is an infrasoft open set $(F, A)$ in $\tau$ such that $F(a)=g^{-1}(U)$. Since $\tau$ generated from crisp infratopologies, we can write $F\left(a^{\prime}\right)=\varnothing$ for each $a^{\prime} \neq a$. Now, $(F, A)=g_{\varphi}^{-1}(H, B)$, where $H(\varphi(a))=U$ and $H(b)=\varnothing$ for each $b \neq \varphi(a)$. By hypothesis, $(H, B)$ is infrasoft open set in $\theta$. Thus, $U$ is infrasoft open set in $\theta_{\varphi(a)}$. Hence, the proof is complete.

The following example shows that a condition imposed on the infrasoft topology of the domain is indispensable.

Example 5. Let $\theta=\{\Phi, \tilde{Y}\}$ be an infrasoft topology on $Y=$ $\{x, y\}$ with a set of parameters $B=\left\{b_{1}, b_{2}\right\}$, and let $\tau=\left\{\Phi, \widetilde{X},\left(H_{1}, A\right),\left(H_{2}, A\right)\right\}$ be an infrasoft topology on $X=$ $\{u, v, w\}$ with a set of parameters $A=\left\{a_{1}, a_{2}\right\}$, where

$$
\begin{aligned}
& \left(H_{1}, A\right)=\left\{\left(a_{1},\{u\}\right),\left(a_{2},\{v\}\right)\right\}, \\
& \left(H_{2}, A\right)=\left\{\left(a_{1},\{v\}\right),\left(a_{2}, \varnothing\right)\right\} .
\end{aligned}
$$

Consider a soft mapping $g_{\varphi}:(X, \tau, A) \longrightarrow(Y, \theta, B)$, where $g: X \longrightarrow Y$ and $\varphi: A \longrightarrow B$ are defined as follows:

$$
\begin{aligned}
g(u) & =x, \\
g(v) & =g(w)=y, \\
\varphi\left(a_{1}\right) & =b_{1}, \\
\varphi\left(a_{2}\right) & =b_{2} .
\end{aligned}
$$

It is clear that $g_{\varphi}$ is a quotient infrasoft mapping. Now, we have $\tau_{a_{1}}=\{\varnothing, X,\{u\},\{v\}\}$ and $\tau_{a_{2}}=\{\varnothing, X,\{v\}\}$ are two parametric infratopologies on $X$, and $\theta_{b_{1}}=\theta_{b_{2}}=\{\varnothing, Y\}$ is a parametric infratopology on $Y$. On the contrary, $g:\left(X, \tau_{a_{1}}\right) \longrightarrow\left(Y, \theta_{\varphi\left(a_{1}\right)=b_{1}}\right)$ is not a quotient inframapping because $g^{-1}(\{x\})=\{u\}$ is an infrasoft open set in $\tau_{a_{1}}$ in spite of $\{x\}$ is not an infrasoft open set in $\theta_{b_{1}}$.

\section{Conclusion}

We study some extensions of soft topology, which are defined by reducing the stipulations of soft topology, for various purposes such as obtaining appropriate models to handle some real-life issues, or building some paradigms that demonstrate the relations among some topological notions and ideas, or keeping certain properties under fewer conditions of those given on soft topology. To this end, we have recently defined a new generalization of soft topology, namely, infrasoft topology.

The principal focus of the article was revising the definition of soft mappings and studying some types of soft mapping in the frame of infrasoft topological structures. The main contributions of this paper are listed as follows.

(1) Improve the definition of soft mapping given in [9] using soft points

(2) Introduce the concepts of infrasoft open, infrasoft closed, and infrasoft homeomorphism mappings, and study several properties with the help of examples

(3) Present the concepts of quotient infrasoft topology and quotient infrasoft mappings and investigate main features

To complete building the infrasoft topological structure, we plan to do the following studies in the frame of infrasoft topological spaces.

(1) Define some types of separation axioms and show the relationships among them

(2) Explore the concepts of compactness and Lindelöffness and establish main characterizations

(3) Initiate the concept of connectedness and research fundamental properties

\section{Data Availability}

No data were used to support the findings of this study.

\section{Conflicts of Interest}

The author declares that there are no conflicts of interest.

\section{References}

[1] D. Molodtsov, "Soft set theory-first results," Computers \& Mathematics with Applications, vol. 37, no. 4-5, pp. 19-31, 1999.

[2] T. M. Al-shami and M. E. El-Shafei, "Partial belong relation on soft separation axioms and decision-making problem, two birds with one stone," Soft Computing, vol. 24, no. 7, pp. 5377-5387, 2020.

[3] T. M. Al-shami and M. E. El-Shafei, "T-soft equality relation," Turkish Journal of Mathematics, vol. 44, no. 4, pp. 1427-1441, 2020.

[4] N. Cağman and S. Enginoglŭ, "Soft matrix theory and its decision making," Computers \& Mathematics with Applications, vol. 59, pp. 3308-3314, 2010.

[5] F. Karaaslan, "Soft classes and soft rough classes with applications in decision making," Mathematical Problems in Engineering, vol. 2016, Article ID 1584528, 11 pages, 2016.

[6] S. Yuksel, T. Dizman, G. Yildizdan, and U. Sert, "Application of soft sets to diagnose the prostate cancer risk," Journal of 
Inequalities and Applications, vol. 2013, no. 1, 229 pages, Article ID x2013-229, 2013.

[7] M. Shabir and M. Naz, "On soft topological spaces," Computers \& Mathematics with Applications, vol. 61, no. 7, pp. 1786-1799, 2011.

[8] N. Cağman, S. Karataş, and S. Enginoglŭ, "Soft topology," Computers \& Mathematics with Applications, vol. 62, pp. 351-358, 2011.

[9] A. Kharal and B. Ahmad, "Mappings on soft classes," New Mathematics and Natural Computation, vol. 7, no. 3, pp. 471-481, 2011.

[10] P. Majumdar and S. K. Samanta, "On soft mappings," Computers \& Mathematics with Applications, vol. 60, no. 9, pp. 2666-2672, 2010.

[11] İ. Zorlutuna and H. Çakır, "On continuity of soft mappings," Applied Mathematics \& Information Sciences, vol. 9, no. 1, pp. 403-409, 2015.

[12] M. E. El-Shafei, M. Abo-Elhamayel, and T. M. Al-shami, "Partial soft separation axioms and soft compact spaces," Filomat, vol. 32, no. 13, pp. 4755-4771, 2018.

[13] T. M. Al-shami and L. D. R. Kočinac, "The equivalence between the enriched and extended soft topologies," Applied and Computational Mathematics, vol. 18, no. 2, pp. 149-162, 2019.

[14] L. D. R. Kočinac, T. M. Al-shami, and V. Çetkin, "Selection principles in the context of soft sets: menger spaces," Soft Computing, 2021.

[15] S. A. El-Sheikh and A. M. Abd El-Latif, "Decompositions of some types of supra soft sets and soft continuity," International Journal of Mathematics Trends and Technology, vol. 9, no. 1, pp. 37-56, 2014.

[16] J. Thomas and J. S. John, "On soft generalized topological spaces," Journal of New Results in Science, vol. 4, pp. 1-15, 2014.

[17] A. H. Zakari, A. Ghareeb, and S. Omran, "On soft weak structures,” Soft computing, vol. 21, pp. 2553-2559, 2017.

[18] T. M. Al-shami, "New soft structure: infra soft topological spaces," Mathematical Problems in Engineering, vol. 2021, Article ID 3361604, 12 pages, 2021.

[19] T. M. Al-shami, "Infra soft compact spaces and application to fixed point theorem," Journal of Function Spaces, vol. 2021, Article ID 3417096, 9 pages, 2021.

[20] T. M. Al-shami and E.-S. A. Abo-Tabl, "Connectedness and local connectedness on infra soft topological spaces," Mathematics, vol. 9, no. 15, p. 1759, 2021.

[21] P. K. Maji, R. Biswas, and A. R. Roy, "Soft set theory," Computers \& Mathematics with Applications, vol. 45, no. 4-5, pp. 555-562, 2003.

[22] S. Nazmul and S. K. Samanta, "Neighbourhood properties of soft topological spaces," Annals of Fuzzy Mathematics and Informatics, vol. 6, no. 1, pp. 1-15, 2013.

[23] T. M. Al-shami, "Comments on some results related to soft separation axioms," Afrika Matematika, vol. 31, no. 7, pp. 1105-1119, 2020.

[24] M. I. Ali, F. Feng, X. Liu, W. K. Min, and M. Shabir, "On some new operations in soft set theory," Computers \& Mathematics with Applications, vol. 57, no. 9, pp. 1547-1553, 2009.

[25] F. Feng, C. Li, B. Davvaz, and M. I. Ali, "Soft sets combined with fuzzy sets and rough sets: a tentative approach," Soft Computing, vol. 14, no. 9, pp. 899-911, 2010.

[26] P. Zhu and Q. Wen, "Operations on soft sets revisited," Journal of Applied Mathematics, vol. 2013, Article ID 105752, 7 pages, 2013.
[27] K. V. Babitha and J. J. Sunil, "Soft set relations and functions," Computers \& Mathematics with Applications, vol. 60, no. 7, pp. 1840-1849, 2010.

[28] J. L. Kelley, General Topology, D. Van Nostrand Company, Inc, Princeton, NJ, USA, 1965. 